\title{
Tales of the Pandemic
}

\author{
Jeffrey M. Weinberg, MD
}

$\Lambda$

fter learning about coronavirus disease 2019 (COVID-19) on the news, we were all aware that it would eventually affect our lives and our dermatology practices. However, once the COVID-19 pandemic arrived in the United States, we were under a shelter-inplace order, schools were shut, and most businesses were closed within a few weeks.

As dermatologists, we were considered essential workers, and our offices could remain open. However, as the numbers of cases accelerated in New York City-the global epicenter of the pandemic — and we approached our peak, I closed down my practice, except for emergencies.

One of the first medical challenges dermatologists faced in the early days of the COVID-19 pandemic was the proper management of our psoriasis patients. The major concern was that patients on biologics and other immunomodulatory therapies might be at an increased risk for COVID-19 infection and increased morbidity if affected. I received a multitude of telephone calls from patients taking these therapies who expressed high levels of concern and anxiety and were looking for direction as to whether they should continue their medications.

Early on, several of our professional societies provided guidelines regarding the use of systemic immunosuppressive agents during the COVID-19 pandemic. On April 15, 2020, the American Academy of Dermatology (AAD) advised, "Dermatologists must delicately balance the risk of immunosuppression with the risk of disease flare requiring urgent intervention with patient-specific risks." ${ }^{11}$ The AAD strongly recommended that patients should not stop their ongoing systemic immunosuppressive therapy without consulting their physicians. The AAD's guidance provided specific recommendations for the following groups: (1) patients on systemic immunosuppressive agents who have not tested positive or exhibited signs/symptoms of COVID-19, (2) patients on systemic immunosuppressive agents who have tested positive for COVID-19 or exhibit signs/symptoms of COVID-19, (3) patients who have halted systemic immunosuppressive therapy after testing positive for COVID-19 (in whom it recommended physicians could reinitiate treatment), and (4) patients being considered for systemic immunosuppressive agents. ${ }^{1}$

The National Psoriasis Foundation (NPF) also recognized the need for additional guidelines for health care providers and patients on managing psoriatic disease during the COVID-19 pandemic. In June 2020, the NPF formed a COVID-19 Task Force, which released its own recommendations for adult and pediatric patients with psoriatic disease. ${ }^{2}$ Similar to the AAD, the NPF COVID-19 Task Force recommended that patients do not stop biologic or oral therapies for psoriasis during the current health crisis, stating the following: "While some uncertainties remain, initial data suggest that the benefit of continuing treatments for psoriatic diseases outweighs the hypothetical risks associated with immune modulating treatment of poor COVID-19-related outcomes for most patients." Individuals in high-risk groups were advised to consult their health care providers regarding whether they should continue or alter therapy during the pandemic, and the clinical decision would be guided by the specific treatment regimen; the patient's age, disease characteristics, and underlying medical conditions; or any particular concerns. Additionally, the task force emphasized that patients with psoriatic disease should continue to follow common sense measures to lower the risk of becoming infected with COVID-19, including practicing physical distancing, wearing face coverings in public settings, and washing their hands regularly. ${ }^{2}$

We remain in the midst of the COVID-19 pandemic with no true guidance as to the future course and impact of the infection. It is important to realize that our understanding of the coronavirus and its impact on our patients is constantly evolving. I encourage all providers to stay current with updates on clinical guidelines. In addition, we should pay attention to the myriad of clinical trials and registries now underway, as they may provide more insight into optimal clinical management in these challenging times.

Most importantly, stay safe!

\section{REFERENCES}

1. American Academy of Dermatology. Guidance on the use of medications during COVID-19 outbreak. https://assets.ctfassets .net/1ny4yoiyrqia/PicgNuD0IpYd9MSOwab47/5e6d85324e7b5aa fed45dde0ac4ea21e/Guidance_on_medications_AHTF_approved _April_15.pdf. Updated April 15, 2020. Accessed July 27, 2020.

2. National Psoriasis Foundation. NPF forms COVID-19 Task Force. https://www.psoriasis.org/advance/coronavirus. Updated July 7, 2020. Accessed July 27, 2020.

From the Department of Dermatology, Ichan School of Medicine at Mount Sinai, New York, New York.

The author reports no conflict of interest.

Correspondence: Jeffrey M. Weinberg, MD, 10 Union Square E, Ste 3C, New York, NY 10003 (jmw27@columbia.edu).

doi:10.12788/cutis.0032 